\title{
Highly Efficient Optimization Mesh Movement Method Based on Proper Orthogonal Decomposition
}

\author{
A. E. J. Bogaers ${ }^{1}$, S. Kok ${ }^{2}$, , , A. G. Malan ${ }^{3}$ \\ ${ }^{1}$ Department of Mechanical and Aeronautical Engineering, University of Pretoria, \\ Pretoria 002, South Africa \\ ${ }^{2}$ Advanced Mathematical Modelling, CSIR Modelling and Digital Science, \\ P.O. Box 395, Pretoria 0001, South Africa \\ ${ }^{3}$ Aeronautic System Competency, CSIR Defense, Peace, Safety and Security, \\ P.O. Box 395, Pretoria 0001, South Africa
}

\begin{abstract}
In this paper, we implement the method of Proper Orthogonal Decomposition (POD) to generate a reduced order model (ROM) of an optimization based mesh movement scheme. In the study it is shown that POD can be used effectively to generate a ROM, that accurately reproduces the full order mesh movement algorithm, with a decrease in computational time of over $99 \%$. We further introduce a novel training procedure whereby the POD models are generated in a fully automated fashion. The technology is applicable to any mesh movement method and enables potential reductions of up to four orders of magnitude in mesh movement related costs. The proposed model can be implemented without having to pre-train the POD model, to any fluid-structure interaction code with an existing mesh movement scheme.
\end{abstract}

\section{INTRODUCTION}

The numerical simulation of flow across a boundary arises in many engineering related problems, e.g. flutter simulations of wings, blood flow through veins and arteries and parachute dynamics. These, and other fluid-structure interaction problems, involve flow induced moving boundaries, and in order to accurately complete these unsteady flow simulations it becomes necessary for the computational grid to conform to the new displaced domain.

In ensuring a boundary conforming mesh, the obvious choice would be to simply regenerate the mesh at each time step. To regenerate the mesh of a complex geometry requires the use of an automatic mesh generator which is an expensive task, especially for three-dimensional problems. The cost can to a certain extent be mitigated by restoring grid quality through localized grid coarsening and refinement [1]. By altering or regenerating a mesh the grid connectivity information changes, and it requires that the solution be projected from the old mesh to the new one. Not only can this be a computationally expensive process, but may lead to conservation issues arising within the fluid domain [2]. As such, a method or algorithm is desired to displace the given mesh to conform to a dynamically moving boundary in a truly computationally efficient manner.

Several algorithms have been developed that can effectively adapt unstructured meshes to the 
new displaced boundary without changing the grid connectivity. This is referred to as mesh movement, examples of which include the spring analogy [3], solving a set of Laplacian or Bi-harmonic equations [4], radial basis function (RBF) interpolation [5, 6] or through mesh optimization [7, 8]. Despite the successes of these algorithms in reducing the frequency and necessity for re-meshing, they may still account for a significant percentage of CPU time for a FSI simulation involving large complex boundary deformations [9].

The aim of this paper is to address the computational cost deficiency of typical mesh movement strategies via the use of effective reduced order models. This is to be done such that the quality of the resulting meshes remains comparable to the original mesh movement methods. The reduced order modeling technique that we make use of is the method of Proper Orthogonal Decomposition (POD), also commonly referred to as Principle Component Analysis (PCA), Singular Value Decomposition (SVD) or Karhunnen-Love (KL) decomposition.

We implement and test the reduced order modelling method to an optimization mesh movement scheme. Furthermore, a novel training procedure is introduced whereby the POD models are generated in a fully automated fashion. The technology is applicable to any mesh movement method and enables potential reductions of up to four orders of magnitude in mesh movement related costs. The proposed model can be implemented without having to pre-train the POD model, to any fluid-structure interaction code with an existing mesh movement scheme.

\section{Optimization Based Mesh Movement}

In this paper we focus the discussion of POD application to optimization mesh movement schemes only. Of the various available mesh movement techniques, mesh optimization arguably produces the highest quality meshes. It is also the only mesh movement scheme that offers direct control over element qualities via the selection of an objective cost function, which in some form describes mesh quality. Despite the ability to optimally deform large unstructured grids, mesh optimization has remained unpopular primarily because of the high computational cost. The associated cost is often prohibitively expensive (of order equal to a full CFD solution) to be used in an actual FSI simulation, making the method a prime candidate to which to apply and demonstrate the reduced order modelling concepts.

Mesh movement through mesh quality optimization is not so much a mesh movement technique but rather a mesh smoothing operation. At each instance of domain boundary movement, the mesh is regularized or smoothed by allowing each of the mesh vertices to relocate, through the use of some optimization algorithm. The mesh is optimized according to an objective function which in some fashion describes the overall quality of the elements. Formally, the mathematical optimization problem is stated as follows:

$$
\underset{\text { w.r.t } \boldsymbol{x}}{\operatorname{minimize}} F(\boldsymbol{x}), \boldsymbol{x}=\left[x_{1}, x_{2}, \ldots, x_{n}\right]^{T}
$$

where $F(\boldsymbol{x})$ is the objective function to be optimized and $\boldsymbol{x}$ is the $x$ and $y$ coordinates of the interior mesh vertices that are allowed to move.

For this particular study we limit ourselves to $2 \mathrm{D}$ unstructured triangular meshes, where the mesh quality is defined by the shape-size metric

$$
F=\sum_{\text {elements }}\left(\frac{r_{\text {out }}}{r_{\text {in }}}\right)\left(\frac{1}{f_{\text {size }}}\right),
$$


where $r_{\text {out }}$ and $r_{\text {in }}$ are the circumscribed and inscribed circles of a triangle respectively, and the quotient $\left(\frac{r_{\text {out }}}{r_{\text {in }}}\right)$ is a measure of the element shape. Assuming isotropic physics, the perfect triangular element shape is an equilateral triangle, for which the quotient is 3 , and tends to infinity as the element deteriorates. $f_{\text {size }}$ is a size metric that relates the volume of an element in its deformed state, $w_{d}$, to the original volume in the starting mesh, $w_{o}$, defined by

$$
f_{\text {size }}=\frac{2 \tau}{1+\tau^{2}},
$$

where $\tau=\frac{w_{d}}{w_{o}}$. The range of $f_{\text {size }}$ is $0 \leq f_{\text {size }} \leq 1$, where 1 implies an exact match, and the quotient $\left(\frac{1}{f_{\text {size }}}\right)$ ranges between 1 and $\infty$ for perfect and degenerate elements respectively. The quality metric (2) is a combination of metrics proposed by Braess et al. [8] and Knupp [10].

The quality metric defined in (2) has one drawback. While it performs well as an optimization cost function, the quality metric itself is not very useful for evaluating post deformation mesh qualities. The values produced by the metric are typically very large, and have little meaning save that they should be as low as possible. As such, for all comparisons and plotting of mesh qualities we make use of the quality metric $f_{\mathrm{ss}}$ defined by Knupp [10], where $f_{\mathrm{ss}}$ ranges between 0 and 1 for degenerate and perfect elements respectively. Because the extremes of the metric are well defined, they allow for easy and intuitive comparisons between respective meshes. The quality metric $f_{\mathrm{ss}}$ is a dimensionless quantity defined by the weighted product

$$
f_{\mathrm{ss}}=\sqrt{f_{\text {size }}} f_{\text {shape }}
$$

where $f_{\text {size }}$ and $f_{\text {shape }}$ denote metrics associated with the size and shape respectively. $f_{\text {size }}$ is square rooted as changes in element size has a smaller influence on mesh quality in comparison to distorted elements.

While allowing for intuitive comparisons, metric $f_{\mathrm{ss}}$ is ill-suited as a cost function in an optimization algorithm. The extremes are not harsh enough and letting a few elements become degenerate will not have a significant impact on the total cost function. For this reason we make the distinction of using the metric defined in (2) as the cost function for the optimization and $0 \leq f_{\mathrm{ss}} \leq 1$ for all post mesh deformation analysis.

For the purposes of this paper, the optimization problem defined in equation (1) is solved using two popular gradient based optimization algorithms, namely the conjugate gradient method and Newton's method with line search [11]. A line search is implemented in conjunction with Newton's method to improve the solution stability. The line search used for both the conjugate gradient and Newton's methods is the standard form of the Powell quadratic interpolation method [11]. The quality metric defined in (2) is continuous and differentiable everywhere, allowing for the first derivatives and the Hessian required by the optimization algorithms to be computed analytically. The algorithms are implemented in MATLAB, and the matrix problem involved in Newton's method is solved using a direct sparse solver to take advantage of the sparsity of the Hessian matrix. Furthermore, LU factorization is used along with the reverse Cuthill-McKee node re-numbering scheme [12] to produce LU factors with near minimal bandwidth.

\subsection{Test Problem}

To demonstrate mesh optimization with application to mesh movement, let us consider a simple test problem involving extreme translation and rotation. The test problem consists of a $200 \times 200$ unit 
square domain with a small inner rectangle that is rotated $60^{\circ}$ counter-clockwise and translated by $\triangle x=30$ and $\triangle y=30$ units, with the initial mesh shown in Figure 1(a). The gray-scale used is for an element shape-size quality metric defined in [10], where $0 \leq f_{\mathrm{ss}} \leq 1$, for degenerate and perfect elements respectively.

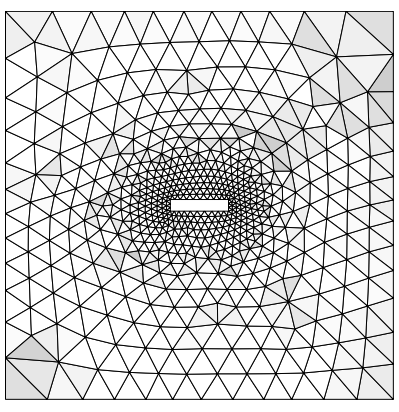

(a)

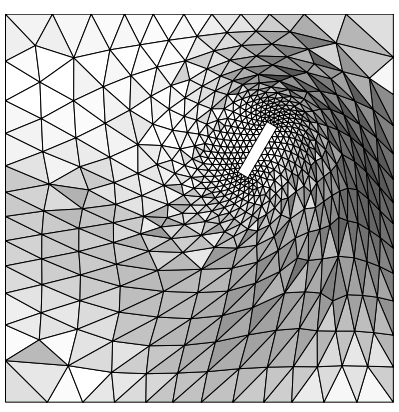

(c)
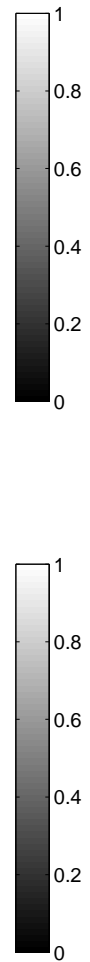

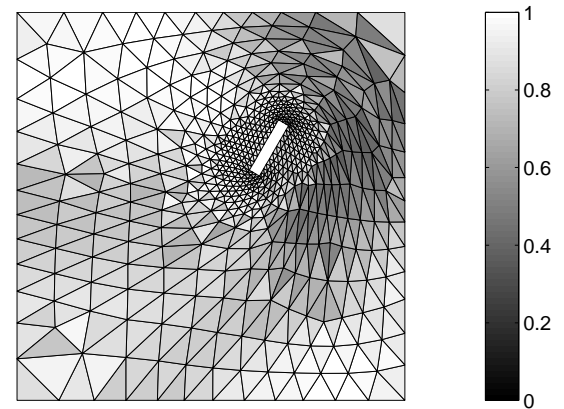

(b)

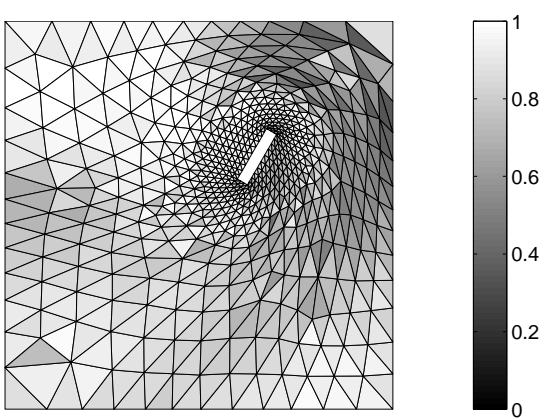

(d)

Figure 1: Comparison of initial and final meshes. (a) Initial mesh, generated using NETGEN [13], (b) Mesh motion using optimization, (c) RBF interpolation and (d) elastic deformation for inner rectangle motion of $\triangle x=30, \triangle y=30, \phi=60^{\circ} \mathrm{CCW}$. Mesh quality gray-scale plots using quality metric $0 \leq f_{\mathrm{ss}} \leq 1$.

Figure 1(b) depicts the resulting mesh of the rotation and translation test. To allow for a comparison, we further show the results using RBF interpolation in Figure 1(c) and elastic deformation in Figure 1(d). RBF interpolation is currently one of the more popular mesh movement methods available, as it results in comparably good quality meshes, at relatively cheap computational costs. For the sake of brevity, we wont discuss the RBF method in detail, but for more information refer to de Boer et al. [5] or Rendall et al. [6]. RBF interpolation moves the internal mesh nodal coordinates according to an interpolation function based on the motion of the boundary nodes. The $\mathrm{RBF}$ interpolation is performed using a $\mathrm{CP} C^{2}$ compact support radial basis function with support radius chosen to encompass the entire domain, similar to de Boer et al. [5]. 
The elastic deformation method is implemented using the finite element method solving the set of linear elasticity equations. The elastic deformation method has a tendency to produce entangled meshes for large boundary deformation because of the infinitesimal strain-displacement assumption for the linear elastic constitutive equations. We therefore augment the method by implementing a Jacobian stiffening factor, which allows for the smaller elements within the mesh to be stiffened as described by Stein et al. [14]. A stiffening factor of 1 is used, which was found to produce good results for this particular problem. Both the RBF interpolation and elastic deformation method was implemented with a total of 15 intermediate displacement increments.

Table 1 depicts the resulting mesh qualities for each of the mesh movement methods based on the quality metric, $0 \leq f_{\mathrm{ss}} \leq 1$. It becomes apparent that of the three methods, mesh optimization produces the highest quality meshes. It has the further advantage that the method is inherently path independent, in other words, for a given boundary deformation there exists a unique mesh. Both RBF interpolation, and the elastic deformation method using small strain linear elastic constitutive equations are path dependent, and the mesh can be expected to deteriorate for FSI simulations involving several large boundary deformations [5].

\begin{tabular}{cccc}
\hline No. & Mesh Movement & $\min \left(f_{\text {ss }}\right)$ & $\operatorname{mean}\left(f_{\text {ss }}\right)$ \\
\hline 1 & Opt: $\left(\frac{r_{\text {out }}}{r_{\text {in }}}\right)\left(\frac{1}{f_{\text {size }}}\right)$ & 0.3747 & 0.8306 \\
2 & RBF: CP C & 0.2724 & 0.8125 \\
3 & Elastic Deformation & 0.3157 & 0.8298 \\
\hline
\end{tabular}

Table 1: Comparison of element qualities for the rotation and translation test problem based on element quality metric $0 \leq f_{\mathrm{ss}} \leq 1$.

There are however two major drawbacks of the optimization method. Firstly, for large grids (common in CFD simulations), the procedure is computationally expensive (approximately of the same order as the CFD simulation). Secondly the maximum boundary displacement cannot be larger than the smallest element size along the boundary. Large displacements lead to the inversion of the boundary elements, which will result in the failure of any optimization algorithm to successfully find an optimal and feasible mesh. To circumvent the problem, the total boundary displacement has to be broken into smaller increments, where each increment will entail a full order optimization problem.

In general transient FSI simulations this would rarely be a problem, as the time steps are usually small enough that each successive boundary deformation is small enough that a single displacement increment would suffice. For our application, where the displacements are large, we minimize the associated cost by providing an initial guess using RBF interpolation. For large deformations, using $\mathrm{RBF}$ interpolation on its own requires that the total motion be divided into smaller displacement increments to attain high quality mesh movement. We however use only a single RBF increment to provide an initial, inversion free starting guess, to which we apply the optimization routine to smooth the mesh.

To illustrate the computational cost of the optimization mesh movement method, Figure 2 depicts the CPU scaling comparison of the three movement methods, for a single displacement increment. Despite providing superior mesh qualities, it may be noted that the optimization method (whether using Newton's method or the conjugate gradient method) is orders of magnitude more expensive than both the elastic deformation and RBF interpolation method. It should also be noted that the computational time reported here for $\mathrm{RBF}$ interpolation is for a compact support 


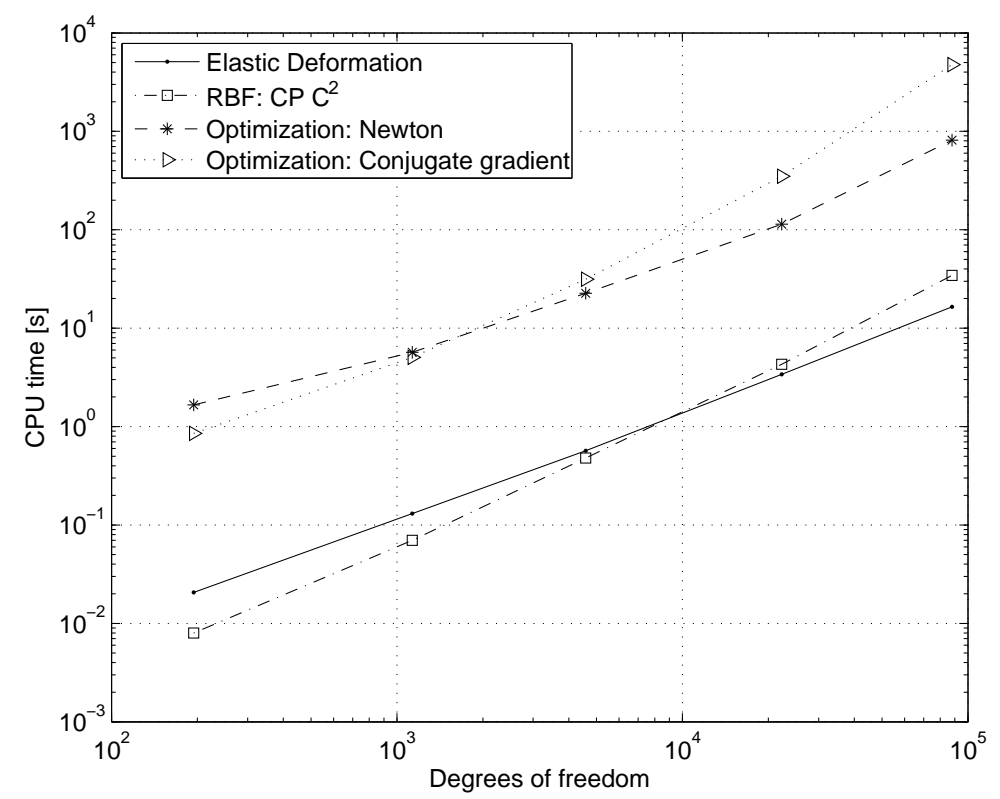

Figure 2: CPU scaling comparison of the three mesh movement methods.

function with fixed support radius. For larger meshes, the support radius can be decreased, which will result in computational speed ups with a minor sacrifice in element qualities. As an example, for the largest mesh in the CPU cost scaling study ( $\mathrm{DOF} \approx 88,000)$ by marginally decreasing the support radius we obtain a $40 \% \mathrm{CPU}$ cost reduction with only an $8 \%$ reduction in mean element quality.

In terms of the two gradient based optimization routines, we find that Newton's method performs better for an increase in problem size. For smaller problems the conjugate gradient method performs better, with a cross over point for a mesh of around 1000 DOF. The cross over point itself will vary from computational platform and implementation, but a mesh of 1000 DOF is still insignificant in comparison to the size of meshes used in actual simulations. For any practical applications, Newton's method would therefore be the preferred optimization method. We highlight the cost comparisons to demonstrate that using a direct method to solve the optimization problem is in fact cheaper than an iterative method. Since the aim of this paper is to demonstrate the ability of POD to speed up mesh movement computations we opt for the cheaper of the two methods to which to make comparisons. It should however be noted that Newton's method is several orders more expensive in terms of memory usage. For the size of problems dealt with in this paper this is not an issue, but should memory become a limiting factor iterative solution schemes such as the conjugate gradient method would be preferred.

Per iteration, the conjugate gradient method is cheaper, but requires a significantly larger number of iterations to reach convergence, and hence the poor CPU cost scaling. The reason for this is based on the manner in which the two methods obtain their respective search directions. The conjugate gradient method only uses first order, local gradient information. As a result, elements far away from one another have no inter-relating information. For example, if the elements along the 
inner rectangle boundary are distorted, the elements further away at the edge of the domain have no gradient information regarding this distortion. It takes several iterations for this information to propagate through the mesh. On the other hand, Newton's method obtains search directions based on a Hessian, which incorporates information for the whole mesh domain. While being more expensive per iteration, Newton's method requires substantially fewer iterations to reach convergence.

\section{POD Based Reduced Order Model of the Optimization Mesh Movement Method}

In this section we aim to introduce and demonstrate the ability of proper orthogonal decomposition (POD) to produce efficient reduced order models of the optimization based mesh movement method.

\subsection{Proper Orthogonal Decomposition}

Proper Orthogonal Decomposition is a mathematical procedure aimed at finding low-dimensional approximate descriptions of high-dimensional systems. POD is in essence an empirical spectral method, similar to Fourier decomposition, where field variables are approximated using expansions of a set of projected basis functions or modes. POD obtains these basis functions from a set of observations, where these observations can be obtained either experimentally or through numerical simulations of a real system. What makes POD remarkable is that the selected modes are not only appropriate but make up the optimal linear basis for describing any given system.

POD has been applied in a wide range of disciplines [15] including image processing, data compression, control in chemical engineering and oceanography. The first use of POD in the field of Fluid Mechanics was by Lumley [16] as a post processing step for determining coherent structures within turbulent flow. Since then POD has successfully been applied to many engineering problems including the characterization of dominant turbulent flow properties [17, 18], aircraft flutter prediction [19] and reduced order models for multidisciplinary optimization [20, 15].

The governing theory, derivation and application of the POD method can readily be found in literature, and for a comprehensive discussion on the method refer to Holmes et al. [17], and to Chatterjee [21] for an easy to understand introduction. In order to keep this paper as self contained as possible, we provide a brief overview of POD, and the method of snapshots.

Suppose we have a set of discrete system observations, or snapshots, $\left\{\boldsymbol{x}^{k}\right\}$ for $k=1,2, \ldots, M$. For mesh movement, these snapshots are the nodal coordinates of the mesh at various instances of boundary displacement. The method of POD extracts the predominant variances of these system observations in the form of optimal linear basis modes $\left\{\boldsymbol{\varphi}_{j}\right\}$, and allows for an approximation of the system observations through the following linear combination:

$$
\boldsymbol{x}^{k} \approx \sum_{j=1}^{M} \alpha_{j}^{k} \boldsymbol{\varphi}_{j},
$$

where $\boldsymbol{\alpha}^{k}$ is an appropriate set of expansion coefficients, relating to the $\mathrm{k}^{\text {th }}$ snapshot $\boldsymbol{x}^{k}$, and $M$ is the number of retained modes.

The POD modes themselves may be computed using the "method of snapshots" developed by Sirovich [22]. The kernel of the POD modes is a finite dimensional autocorrelation matrix of the 
form

$$
\mathcal{R}=\frac{1}{M} \boldsymbol{X} \boldsymbol{X}^{T}
$$

where $\boldsymbol{X}$ is an observation matrix of size $M \times N$, where each row vector of the matrix represents a snapshot. $N$ for a 2D mesh is the total number of $x$ and $y$ nodal coordinates and $M$ is the number of snapshots. The eigenvectors $\boldsymbol{a}$ of $\mathcal{R}$ are computed as an intermediate step from

$$
\mathcal{R} a=\lambda a,
$$

where the POD basis modes can then be computed as the linear combination

$$
\boldsymbol{\varphi}^{k}=\sum_{i=1}^{M} a_{i}^{k} \boldsymbol{x}^{i} \text { for } k=1,2, \ldots, M,
$$

where $a_{i}^{k}$ is the $i^{t h}$ element of eigenvector $\boldsymbol{a}^{k}$ corresponding to $\lambda_{k}$. For the mesh movement problem, the eigenvalues $\lambda$ have no physical interpretation, save that their magnitudes provide an indication as to how much of the system information is captured by the associated POD mode. Ordering $\lambda$ and the associated POD modes in order of descending magnitude, we may reproduce the approximation in (5) by retaining only the first $K$ most dominant modes.

It should also be noted that if the coefficients in (5) are chosen as the eigenvectors $\boldsymbol{a}^{k}$, corresponding to the $k^{\text {th }}$ POD mode, then we would generate an approximation of the $k^{\text {th }}$ snapshot. If however we would like to compute an approximation of the mesh movement based on boundary displacements other than those used in the training snapshots, we would require the computation of an appropriate set of expansion coefficients $\boldsymbol{\alpha}$.

\subsection{Snapshot Generation}

The first requirement for generating a POD model is to acquire a set of snapshots. A POD based model can only reproduce information within close proximity to the information contained in these snapshots. The boundary movements for the representative snapshots must therefore be chosen to closely mimic the expected movement in an actual simulation. This inherently means that certain a priori knowledge is required as to how the actual system will behave. Fortunately, in most engineering problems, a substantial amount of information relating to a problem, such as the predominant physics at play, is usually known, or can be estimated prior to the simulation. We do however in the latter part of the paper introduce an adaptive training methodology that circumvents this problem.

For the purposes of the POD demonstration, let us revisit the test problem introduced in Section 2.1, of the inner rectangle undergoing large rotations and translations. We generate 60 snapshots as a pre-processing step, where the magnitudes of rotation and translation of the inner rectangle is chosen via the method of Latin hypercube sampling (LHS). There is no way of knowing prior to an actual analysis how many snapshots are required to adequately describe the system, and the choice of 60 snapshots is selected rather arbitrarily. LHS is often used in uncertainty analysis, and was developed primarily to generate a distribution of plausible collections of parameter values from a multidimensional distribution [23, 24]. In essence, LHS allows for random selections of the displacement and rotation magnitudes, with uniform distribution. Therefore in theory, because of the uniform distribution of data points, and the fact POD extracts the predominant system 
variances, the model should now contain information covering most combinations of rotations and translations within the sampled region.

The mesh movement for each of the LHS specified locations is performed using the mesh optimization method with an initial guess provided using RBF interpolation, as previously discussed.

\subsection{Approximate Models of Optimization Mesh Movement Using POD}

With the snapshots generated, we are able to compute the POD basis modes, where Figure 3 shows the ordered eigenvalues associated with the POD modes. From the magnitudes of the eigenvalues it may be noted that the first 10 POD modes contain over $99 \%$ of the system information. The error $E$ of retaining only the first $K$ most dominant POD modes may be approximated by

$$
E=1-\frac{\sum_{j=1}^{K} \lambda_{j}}{\sum_{i=1}^{M} \lambda_{i}} .
$$

By retaining only the first 10 modes, the degrees of freedom of the problem is essentially reduced from $2 N$, the total number of coordinates, to just 10 .

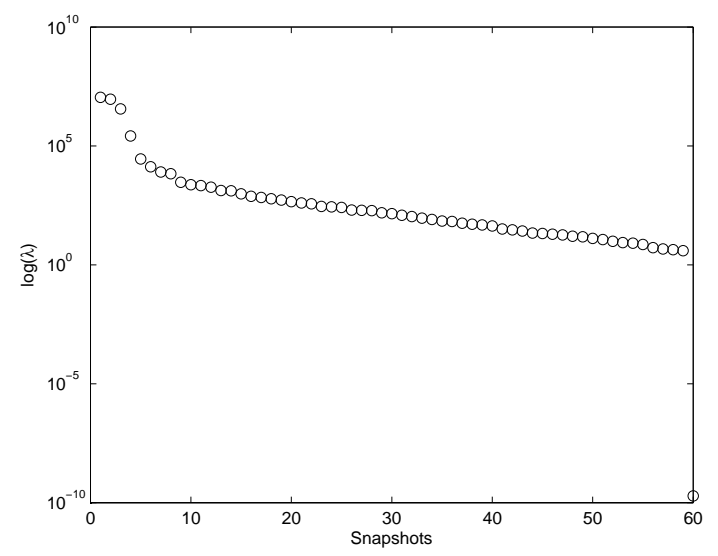

Figure 3: Ordered eigenvalues, which provides an indication of the percentage of the system information that is captured by the associated POD modes.

To illustrate the contributions made by each of the POD basis modes, representations of the first 5 modes are shown in Figure 4. The first two modes appear to contain the predominant system information, and handles translations as well as some rotation information for the inner block movement. The third POD mode contains information with regards to block rotation and expansion, and mode 4 controls the degree of element contractions around the inner block. The type of information contained within modes 5 and upwards cannot be expressed in terms of simplistic motions, however the magnitude of their contributions is minimal in comparison to the first 4 modes. The expansion coefficients $\boldsymbol{\alpha}$ scales the magnitude and directions of each of the POD modes contribution to the overall solution via the approximation in (5), reproduced below for ease: 


$$
\boldsymbol{x}^{k} \approx \sum_{j=1}^{K} \alpha_{j} \boldsymbol{\varphi}_{j}
$$

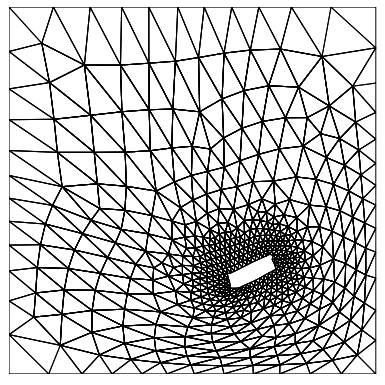

(a) Mode 1

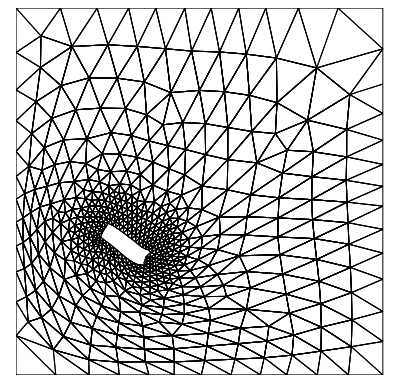

(b) Mode 2

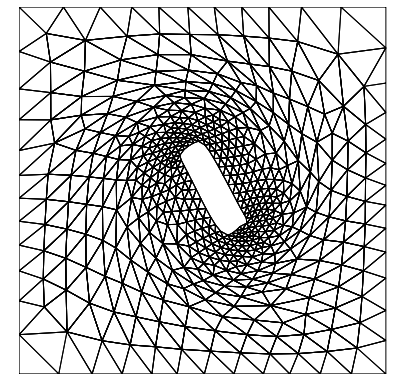

(c) Mode 3

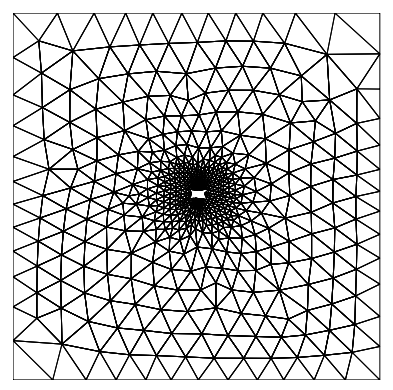

(d) Mode 4

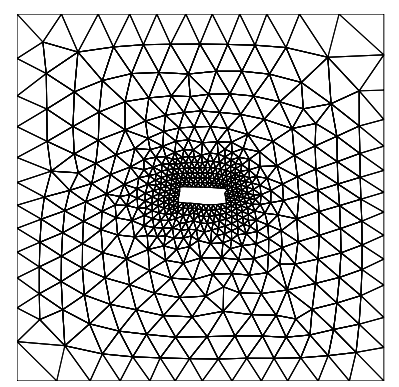

(e) Mode 5

Figure 4: Representations of the first 5 POD modes. Magnification factor of 0.3 .

With the POD modes computed, we can substitute the approximation for $\boldsymbol{x}$ in (10) into (1), allowing for the solution of the original optimization problem, but now only in terms of the unknown expansion coefficients $\boldsymbol{\alpha}$, and the known POD modes $\boldsymbol{\varphi}$,

$$
\underset{\text { w.r.t. } \alpha}{\operatorname{minimize}} F(\boldsymbol{x}(\boldsymbol{\alpha})), \boldsymbol{\alpha}=\left[\alpha_{1}, \alpha_{2}, \ldots, \alpha_{n}\right]^{T} .
$$

There is however a slight problem in attempting to solve the optimization problem using equation (11). The objective function based on $\boldsymbol{\alpha}$ contains a large number of local minima. To illustrate, an example of the function profile $F(\boldsymbol{x}(\boldsymbol{\alpha}))$ is shown in Figure 5, generated by altering $\alpha_{1}$. Because of these local minima, there is no gradient based optimization routine available that will guarantee convergence of the solution to the global minimum, unless the starting points are chosen to be close to the exact solution, or more appropriately, within the valley of the global minimum.

The presence of the local minima is attributed to element inversion. For any instance in which there are elements within the mesh that are inverted a local minimum will exist. In order to optimize the cost function in terms of $\boldsymbol{\alpha}$ an initial inversion free guess is required. We therefore propose the use of an interpolation method to obtain an approximation to the coefficients as an initial guess. Interpolation would be used to equate a function relating the expansion coefficients to the moving boundary coordinates based on information gathered from the generated set of snapshots. 


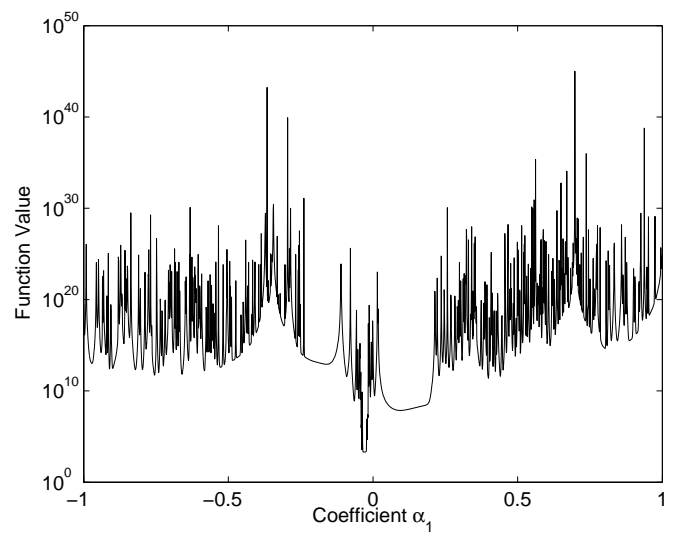

(a)

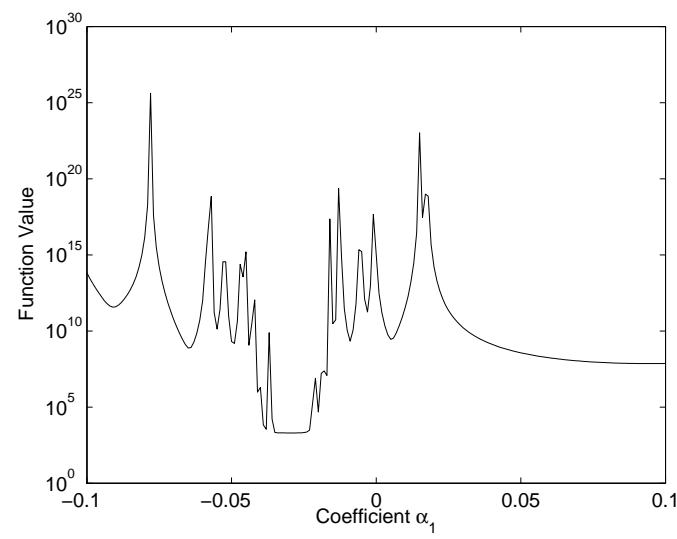

(b)

Figure 5: Example function profile for objective function as a function of expansion coefficient $\alpha_{1}$ with (a) $\alpha_{1} \in[-1 ; 1]$, (b) $\alpha_{1} \in[-0.1 ; 0.1]$

The most commonly used method for generating surrogate models is the method of Least Squares (LS) using some polynomial basis [26]. To demonstrate the ability of interpolation methods to approximate the coefficients we move the inner rectangle along a predefined path. The path is depicted in Figure 6, where the inner rectangle moves from $\triangle x=30, \triangle y=30, \phi=60^{\circ} \mathrm{CCW}$ (position A), through the origin (B) to $\triangle x=-30, \triangle y=-30, \phi=60^{\circ} \mathrm{CCW}$ (C). The approximations of the first 6 expansion coefficients are shown in Figure 7, along with the exact values of the respective expansion coefficients

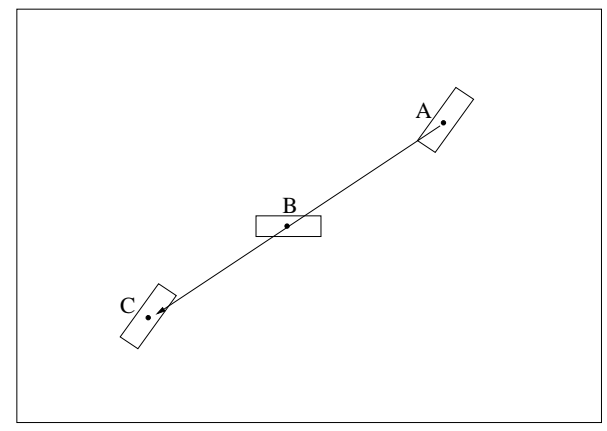

Figure 6: Pre-defined path for the motion of the inner rectangle between $\triangle x=30, \triangle y=30, \phi=$ $60^{\circ} \mathrm{CCW}$ to $\triangle x=-30, \triangle y=-30, \phi=60^{\circ} \mathrm{CCW}$. The expansion coefficients $\boldsymbol{\alpha}$ for the POD model along the path are shown in Figure 7.

From the coefficient plots in Figure 7, it may be noted that the linear LS function adequately approximates the first 4 expansion coefficients. However, from $\alpha_{5}$ onwards, the relationship between boundary positions and expansion coefficients become too non-linear for a linear basis polynomial. This approximation can be significantly improved by using a higher basis polynomial; the approx- 
imations based on a quadratic polynomial are also shown in Figure 7. It is certainly conceivable that using a cubic, or even higher polynomial basis would provide even better approximations.

Using higher order polynomials does however have serious cost implications. For example, for a $2 \mathrm{D}$ mesh, the size of the matrix problem to be solved using a linear polynomial is of size $\left[2 n_{b}+1\right] \times\left[2 n_{b}+1\right]$, where $n_{b}$ is the number of boundary nodes used to generate the approximation. In contrast, using a quadratic polynomial, the matrix problem size increases to $\left[n_{b}^{2}+3 n_{b}+1\right] \times$ $\left[n_{b}^{2}+3 n_{b}+1\right]$, where each matrix problem will have to be solved $K$ times, where $K$ is the number of expansion coefficients to be solved for. The cost implications can however become rather severe. As an example, for a problem with 100 boundary nodes, a linear approximation requires the solution of a $201 \times 201$ linear system, compared to a $10301 \times 10301$ linear system using a quadratic polynomial basis. The associated memory required also becomes prohibitive, considering that the matrices consist entirely of non-zero entries.

The CPU and memory costs are not that severe if one considers that the matrix problem only has to be solved once off, and can be done using an iterative solver. Furthermore, it is not necessary that all the boundary nodes be used in the interpolation function. For example, in our problem with the rectangle, only the corner nodes need be used, as they completely describe the position and orientation of the block. On the other hand, if the boundary surface is more complex, for example an aircraft wing, only every $n^{\text {th }}$ node needs to be accounted for, where $n$ can be changed to include all the nodes or only a small number of key nodes.

Having said that, it would be preferable if an interpolation method is used that can generate relationships between highly non-linear data, that scales better than quadratic LS. Since RBF interpolation has already been used in the preceding part of the work, a fully functioning RBF code is already available, and is hence the interpolation method chosen. The approximations produced using RBF interpolation, with $\mathrm{CP} C^{2}$ radial basis functions, is also shown in Figure 7. RBF provides as good an approximation as 2nd order LS, while the size of the matrix to be solved is only $\left[2 n_{b}+1\right] \times\left[2 n_{b}+1\right]$.

Using RBF interpolation, we now have an effective means by which to approximate the POD coefficients. These approximations can now be used as the initial starting point for the optimization routine. The RBF approximations are in fact so good, that further optimization of the expansion coefficients is in most cases not necessary, unless greater accuracy is desired.

\subsection{Approximation Accuracy of POD Model}

To summarize, at this point we generated 60 snapshots of the mesh at various positions and angles of rotation based on hypercube generated sampling points. The POD basis modes were then computed based on these snapshots. We then suggested two ways to solve the POD coefficients: i) to approximate the coefficients using RBF interpolation, and ii) to use these approximations as an initial starting point for an optimization routine to solve equation (11).

It remains to quantify how well the POD model is able to reproduce the meshes for various boundary motions. The optimization routine that will be made use of for the test is the NewtonRaphson method. The DOF for the ROM is small enough that it will be computationally efficient, and we gain from second order convergence. Secondly, since the starting point for the optimization will be a good one, we are mostly guaranteed of convergence.

To demonstrate the ability of the POD based ROM, the inner rectangle is translated by 30 units in the $x$ and $y$ directions and rotated by $60^{\circ}$ counter clock-wise. The meshes produced by the ROMs for the mesh movement are shown in Figure 8. Note that the POD based ROM is 


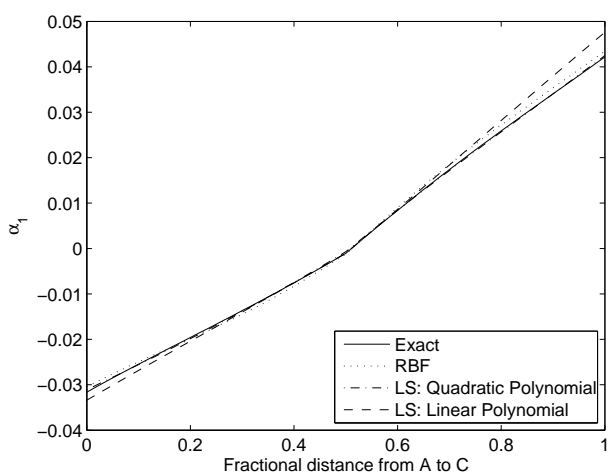

(a)

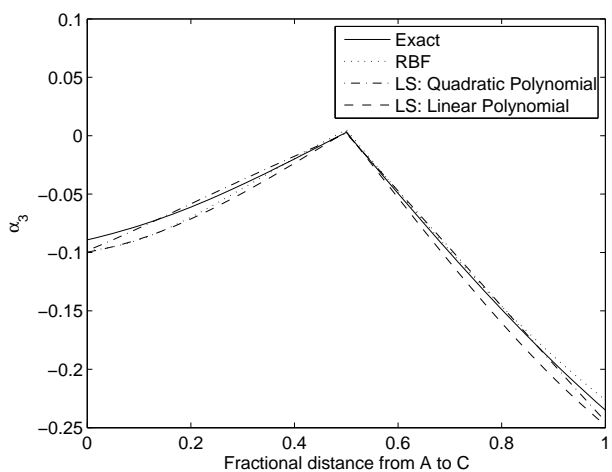

(c)

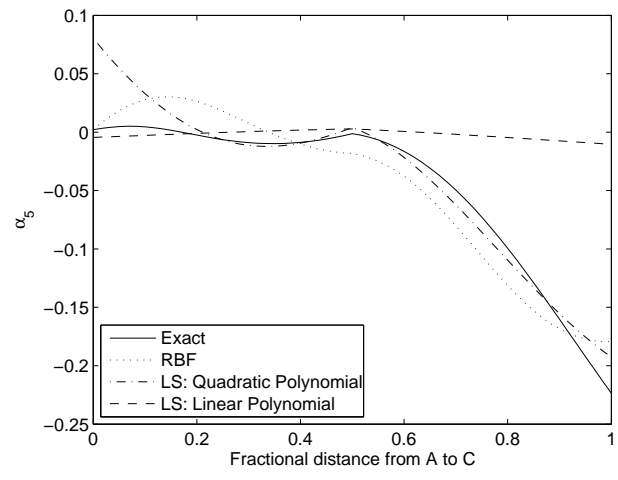

(e)

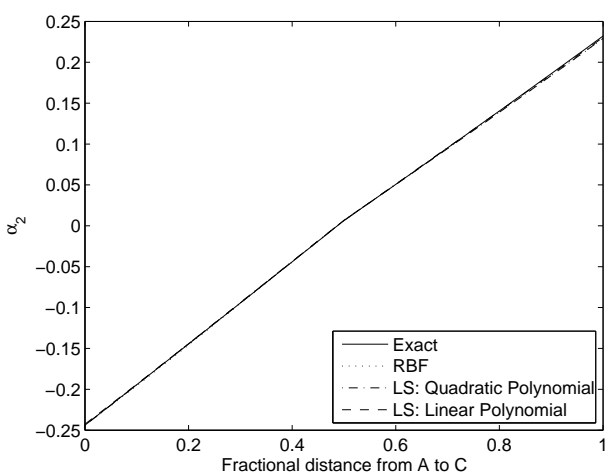

(b)

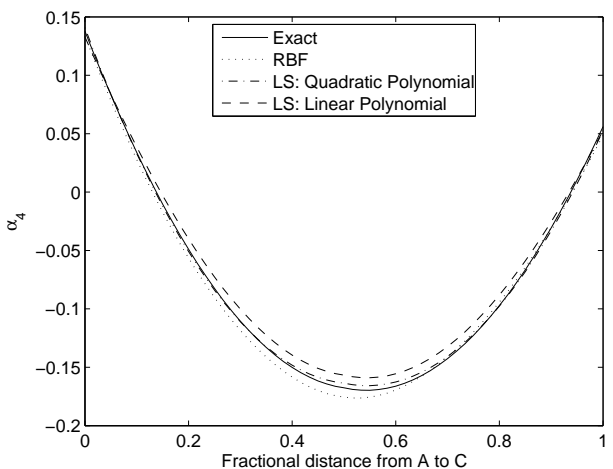

(d)

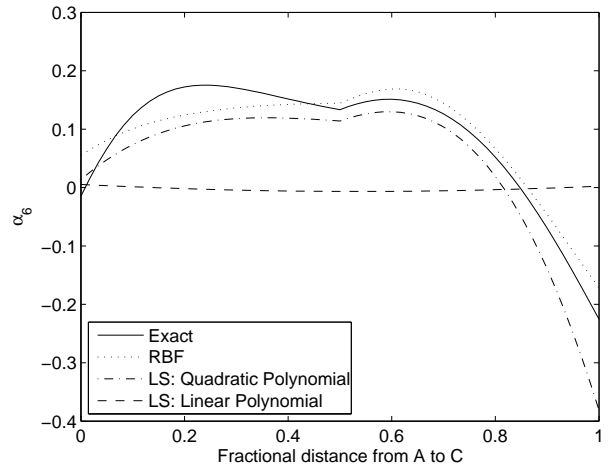

(f)

Figure 7: Plots of the first 6 expansion coefficients $\boldsymbol{\alpha}$ along the predefined path illustrated in Figure 6. (a) $\alpha_{1}$, (b) $\alpha_{2}$, (c) $\alpha_{3}$, (d) $\alpha_{4}$, (e) $\alpha_{5}$ and (d) $\alpha_{6}$.

capable of reproducing the full order mesh movement. Also note that the ROM constructed via $\mathrm{RBF}$ interpolation is in fact very good. 


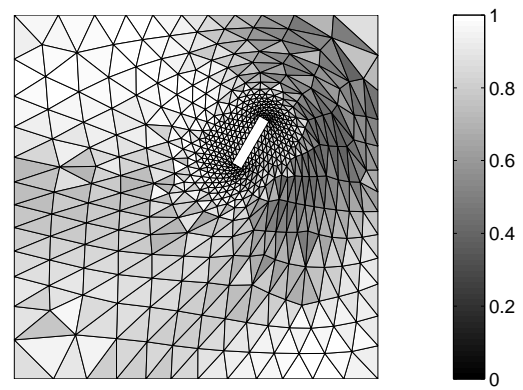

(a) Full order mesh optimization.

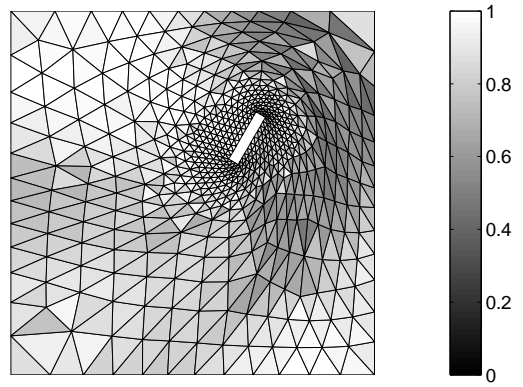

(b) ROM: Coefficient Interpolation.

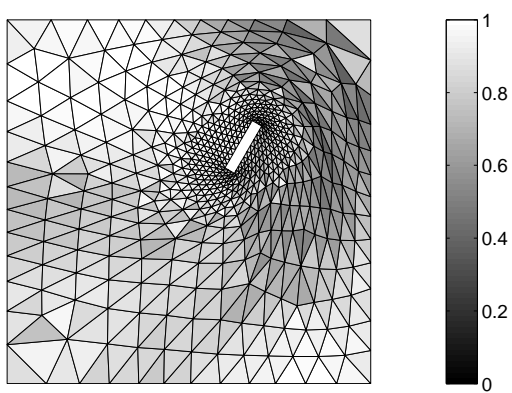

(c) ROM: Coefficient Optimization.

Figure 8: Rotation and translation test case for inner rectangle movement of $\triangle x=30, \triangle y=$ 30 and $\phi=60^{\circ} \mathrm{CCW}$. Mesh quality gray-scale plots based on quality metric $0 \leq f_{s s} \leq 1$ [10].

Comparing the element qualities of the ROM with the full order optimization, we find that using only coefficient interpolation the mean percentage difference in element qualities is $0.3477 \%$, and using Newton's method to further optimize the coefficients we find a difference of $0.0274 \%$. Figure 9 presents histograms comparing the percentage difference in terms of each element's size and shape for the mesh generated by the ROMs and the full order mesh optimization. Using the ROM constructed via RBF interpolation we find that $97.83 \%$ of the elements' size differ by less that $5 \%$ and $86.11 \%$ of the elements' shapes differ by less than $5 \%$. There is only a minor improvement after the use of Newton's method, with $98.68 \%$ and $88.28 \%$ of the elements' size and shape respectively differing by less than $5 \%$. The histograms show that some of the elements experience either a positive or negative size and shape change. The positive changes indicate that the element shape and sizes improved in comparison to the full order optimization. Notice that the histograms are centered about zero, indicating that on average the mesh quality does not deteriorate significantly.

\subsection{Computational Cost and Efficiency of the POD Based ROM}

To compare the computation cost of the ROM, Figure 10 shows the CPU cost scaling for the rotation and translation test problem. 

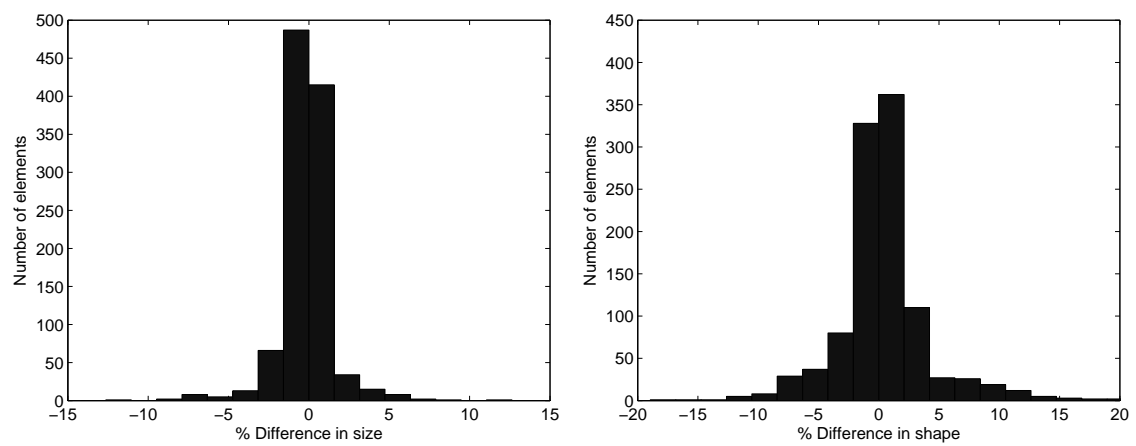

(a) POD ROM with coefficient interpolation only.
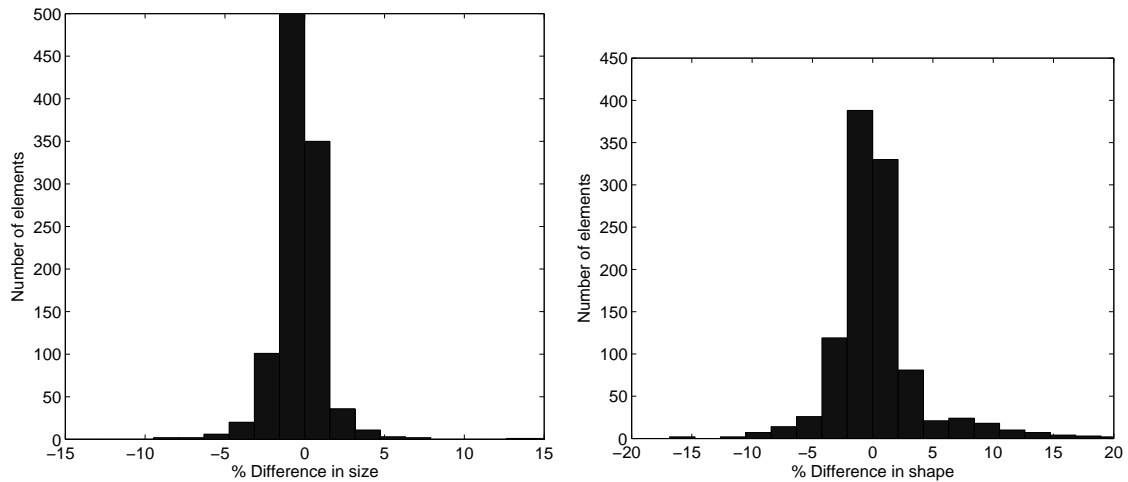

(b) POD ROM with coefficient optimization.

Figure 9: Histogram plot of the percentage difference in shape and size for each element in the mesh of the ROMs compared to the full order mesh optimization. For $\triangle x=30, \triangle y=30, \phi=60^{\circ} \mathrm{CCW}$.

Both ROMs achieve significant CPU cost reduction. If we compare to the full order Newton's method, we find total CPU savings ranging from $87.35 \%$ to $96.51 \%$ for the smallest to largest mesh respectively for the ROM using coefficient optimization. While these cost reductions are noteworthy, even greater savings of up to $99.99 \%$ is found by using interpolation alone, and more importantly a CPU cost scaling factor of 0.032 .

Computing the ROM coefficients through interpolation does not require the solution of any equations, but only evaluating a set of interpolation functions. The cost of these interpolation functions is based only on the number of grid points along the moving boundary and the number of retained POD modes. The number of nodes along the inner boundary is orders less than the total DOF of the mesh, and the number of modes and coefficients is kept constant at 10. As a result, we now have a mesh movement solution methodology, with an associated cost that essentially remains constant as the mesh problem size increases. 


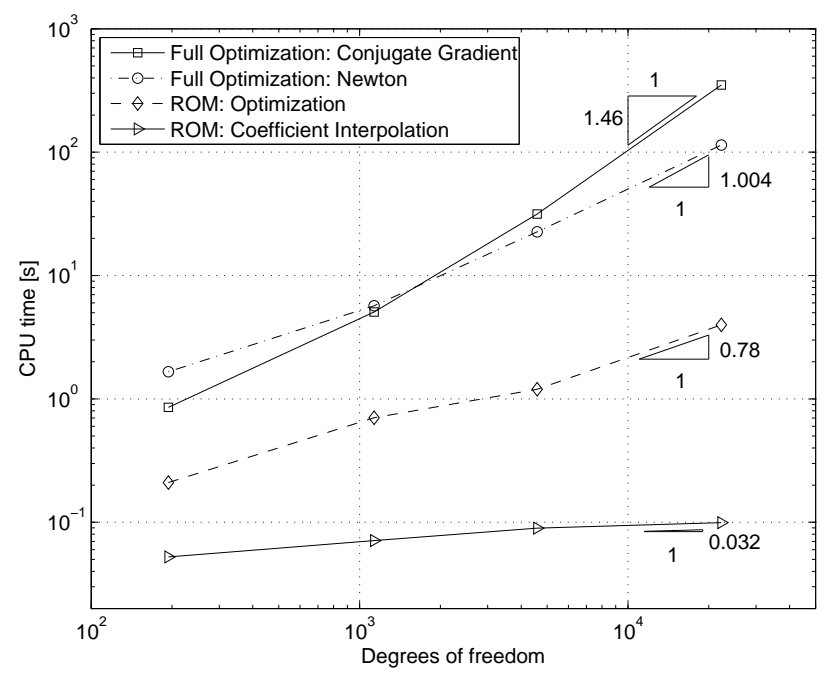

Figure 10: Comparison of the CPU scaling of the ROMs.

\section{Adaptive Training}

In the preceding section we demonstrated that POD can be used to generate an accurate ROM of optimization based mesh movement while effecting significant reductions in computational times. It is fairly hard to justify the use of ROM if it requires pre-training, as would traditionally be necessary for the use of POD. This either means that accurate information with regards to the boundary deformation needs to be known prior to a simulation, or that a full FSI simulation needs to be performed in order to attain this information. If a full FSI simulation is required to generate a POD model of the mesh movement, it would make far more sense to generate a ROM of the full FSI results rather than the mesh movement alone.

On the other hand, it is possible to generate the POD training snapshots based on predictions of the boundary movement. There does however remain the question as to how many training snapshots are required, and how they should be selected. Generating snapshots of hypothetical boundary displacements, without prior knowledge of the simulation, allows for the possibility that erroneous selections are made. The snapshots might not properly define the actual motion, or computational time might be wasted on snapshots that may never feature in the full FSI simulation.

To alleviate these issues, we propose the use of an adaptively trained POD model, to facilitate the full order mesh movement, with the aim of reducing computational costs for first time simulations.

\subsection{Adaptive Training Procedure}

The adaptive training procedure itself is fairly simple. The POD model is initialized using the starting mesh in its undeformed configuration, and the first updated mesh obtained using a full order mesh optimization. The POD model is then used for all subsequent boundary deformations until the mesh quality drops below some lower limit. A full order mesh optimization is then performed to smooth out the mesh, with the results used as an additional snapshot to update the POD model. 
The lower limits themselves do not have to be known beforehand and can also be set adaptively based on information in preceding updating snapshots. In our demonstration we set the lower limits in terms of both the minimum and mean element quality to 10 percent lower than the worst element qualities obtained within any preceding updates. By setting the lower limits adaptively the risk of setting these limits either too high or too low is alleviated.

Mesh optimization is particularly well suited to the adaptive training model because there exists a unique solution for any given boundary movement. At each instance where the mesh movement based on the ROM deteriorates below the lower limits, and an updating snapshot is required, the mesh is regularized by running a full order optimization routine. For path dependent methods such as RBF interpolation, once the mesh has degenerated, running a full order mesh movement step would not improve the quality of the mesh. Having said that, the method is applicable to any mesh movement method, as long as sufficient precautions are taken. For example, if RBF interpolation is used to generate the updating snapshots, the boundary and mesh can be displaced from the original position to the deformed state as long as many incremental displacement steps are used.

The proposed method of POD is fully automated, and is a 'black box' type wrapper that can be applied as is to any FSI code with an existing mesh movement algorithm. It will allow for computational cost reductions of first time simulations, without the user having prior knowledge regarding system behaviors.

\subsection{Adaptive Training Test Case}

To demonstrate the adaptive training procedure, we make use of the common FSI benchmark simulation of flow across a flexible tail member $[27,28]$. The geometry, as well as the initial mesh for the problem is depicted in Figure 11. The FSI simulation is performed using a unified finite volume code currently under development by Oxtoby and Malan [9]. The pressure plots for the beam undergoing predominantly first mode oscillations are shown in Figure 12 with the y-displacements of the beam tip shown in Figure 13. The simulation itself is performed using a total of 4500 time steps to ensure limit state convergence.

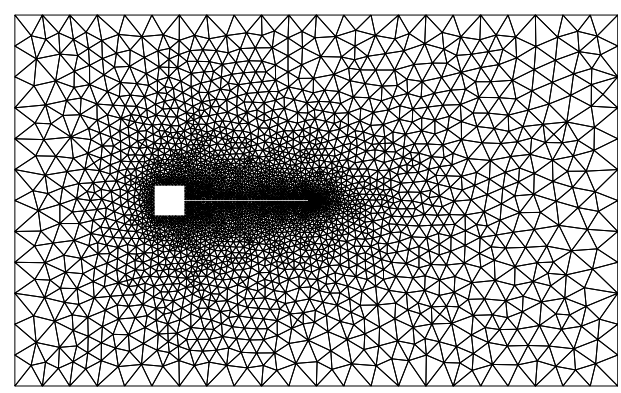

Figure 11: Initial mesh for oscillating tail benchmark problem.

In total, the adaptive model requires only 16 updating snapshots. To provide an indication as to how well the POD ROMs replicate the full order mesh movement, the normalized error function between the exact and approximate nodal coordinates is plotted in Figure 14, where the error function is defined by 

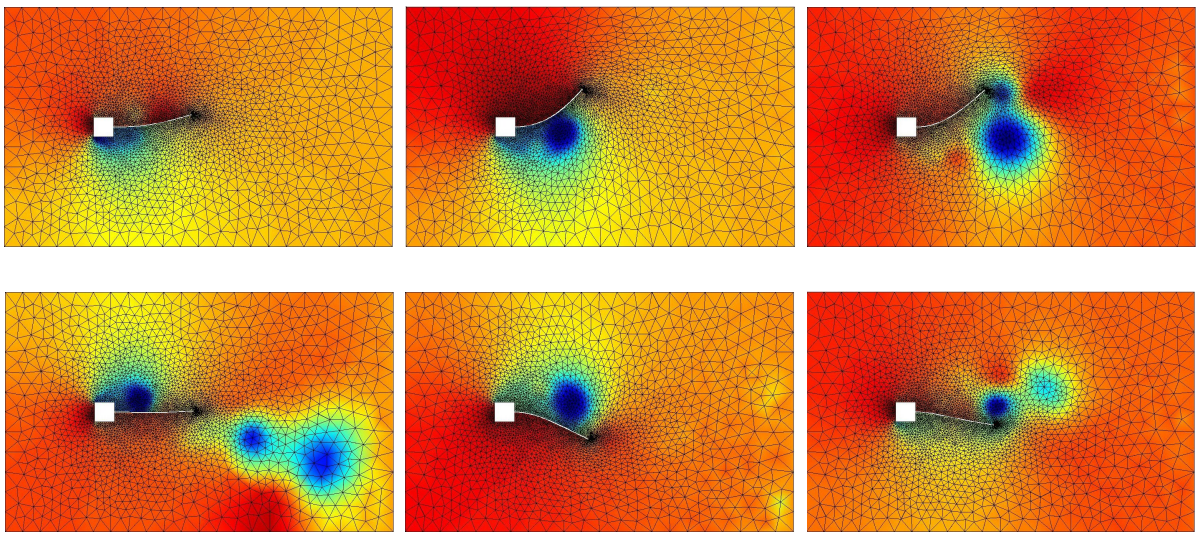

Figure 12: Pressure plots for first mode deformations at selected time steps.

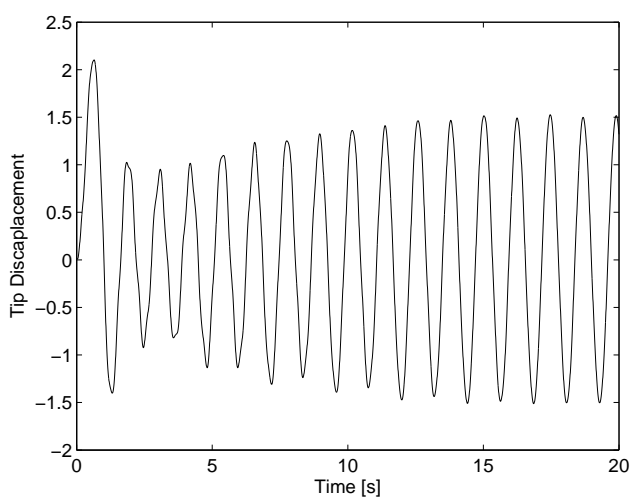

Figure 13: First mode deformation computed using 4500 time steps.

$$
\|e\|_{2}=\frac{1}{N}\left(\frac{\sum_{i=1}^{N}\left\|\boldsymbol{x}_{i}-\boldsymbol{x}_{i_{\text {exact }}}\right\|}{l}\right)
$$

where $l$ is the average length of all edges connected to node $i$ and $N$ is the total DOF of the mesh.

From the plot, it is evident that the ROM based on coefficient optimization outperforms the ROM based on coefficient interpolation. However, of far more importance than the actual differences in nodal coordinates, is whether the meshes generated by the ROMs are still appropriate for use in a simulation. To this end, the mean element qualities for the full mesh optimization and the ROM based only on coefficient interpolation is shown in Figure 15 along with the 16 updating positions. The mean element qualities is based on $0 \leq f_{\mathrm{ss}} \leq 1$.

The adaptively trained model produces near exact approximations of the full order mesh movement. What makes the model really attractive is the comparative computational cost. For the full mesh movement problem, we have a total of 4500 time steps, where each time step takes approximately 22 seconds to solve for the optimization problem. In comparison, the adaptively trained 


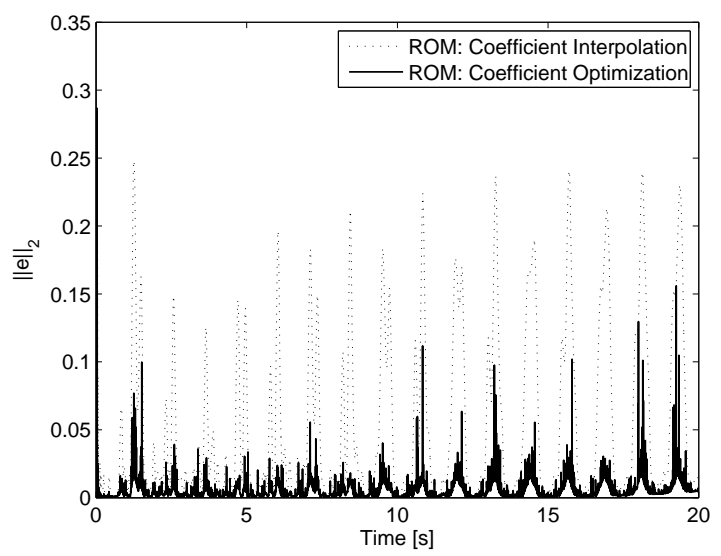

Figure 14: Normalized error between the nodal coordinates obtained by the POD ROMs compared to the full order mesh optimization for the first mode deformation.

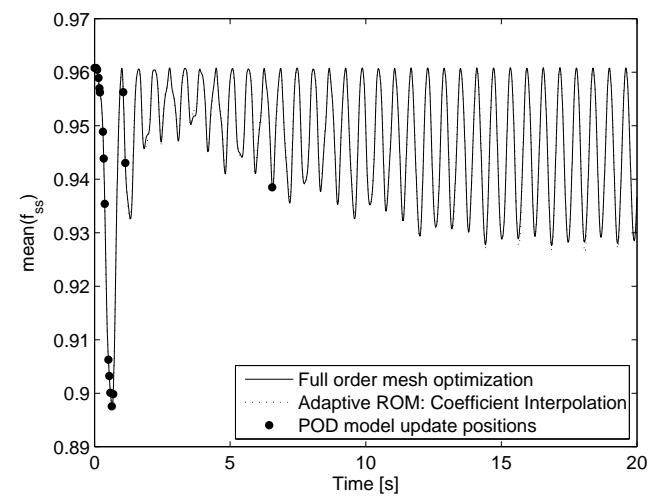

Figure 15: Mean element quality comparison using full order mesh optimization and adaptively trained POD ROM based on coefficient interpolation. A total of 16 updating snapshots are required.

model requires only 16 updating snapshot solutions, where each update would now consist of a full optimization problem. The POD model itself requires only around 13.5 seconds to solve the mesh movement problem for the full 4500 time steps and an additional time of around 3 seconds is required to update the POD model. The total time for the mesh movement problem is hence reduced from close to 27 hours to approximately 400 seconds, or a reduction from 4500 full order mesh optimization time steps to just 16 .

The times reported should not be viewed as definitive as they are based solely on our implementation of the various methods. These times may vary largely based on several factors, of which implementation is but one. We report on the times to provide an indication of potential computational savings when implementing the POD method. It should also be noted that mesh optimization is arguably the most expensive mesh movement method available. The percentage savings that the method offers would be significantly reduced if another mesh movement method is 
used. The POD method itself should also not be confused with a stand alone mesh movement algorithm. It is merely a tool that may be implemented to facilitate the speed up of any conventional mesh movement method.

\section{Conclusion}

In this paper we demonstrated the ability of the method of POD to generate effective reduced order models of mesh movement algorithms. We implemented and tested the method on an optimization mesh movement scheme, though the method itself is just as easily applicable to any other mesh movement method. In the first test case, the POD model was trained as a pre-processing step to a simple combined rotation and translation test case, and it was found that computing the POD expansion coefficients using interpolation alone resulted in comparably good quality final meshes, with CPU cost reductions in excess of $99.99 \%$. We also introduced an adaptive training procedure that allows for the use of the POD method, in conjunction with a full order mesh movement solver, without the ROM having to be pre-trained.

\section{References}

[1] Cavallo P.A., Sinha N., Feldman G.M. Parallel unstructured mesh adaptation for transient moving body and aeropropulsive applications American Institute of Aeronautics and Astronautics $2004 ; \mathbf{1 0 5 7}$

[2] van Loon R, Anderson P.D., van de Vosse F.N., Sherwin S.J. Comparison of various fluidstructure interaction methods for deformable bodies. Computers and Structures 2007; 85:833843.

[3] Farhat C, Degend C, Koobus B, Lesionne M. Torsional springs for two-dimensional dynamic unstructured fluid meshes. Computer Methods in Applied Mechanics and Engineering 1998; 163:231-245.

[4] Helenbrook B. T. Mesh deformation using the biharmonic operator. International Journal for Numerical Methods in Engineering 2003; 56: 1007-1021.

[5] de Boer A, van der Schoot MS, Bijl H. Mesh deformation based on radial basis function interpolation. Computers and Structures 2007; 85:784-795.

[6] Rendall TCS, Allen CB. Unified fluid-structure interpolation and mesh motion using radial basis functions. International Journal for Numerical Methods in Engineering 2008; 74:1519-1559.

[7] Diachin L.F., Knupp P., Munson T., Shontz S. A comparison of two optimization methods for mesh quality improvement. Engineering with Computers 2006; 22(2):61-74.

[8] Braess H, Wriggers P. Arbitrary lagrangian eulerian finite element analysis of free surface flow. Computer methods in applied mechanics and engineering 2000; 190:95-109.

[9] Oxtoby O, Malan AG. A unified finite-volume approach to fluid-structure interaction. In 15th International Conference on Finite Element in Flow Problems (FEF09), Japan, Tokyo, April 2009 
[10] Knupp PM. Algebraic mesh quality metrics for unstructured initial meshes. Finite Element in Analysis and Design 2003; 39:217-241.

[11] Snyman JA. Practical Mathematical Optimization: An Introduction to Basic Optimization Theory and Classical and New Gradient-Based Algorithms. Springer-Verlag, 2005.

[12] George A, Liu J. Computer Solutions of Large Sparse Positive Definite Systems. Prentice-Hall, 1981.

[13] Schberl J. NETGEN An advancing front 2D/3D-mesh generator based on abstract rules. Computing and Visualization in Science 1997; 1(1):41-52.

[14] Stein K, Tezduyar T, Benney R. Mesh moving techniques for fluid-structure interactions with large discplacements Transactions of the ASME 2003; 20:58-63.

[15] LeGresley PA, Alonso JJ. Airfoil design optimization using reduced order models based on proper orthogonal decomposition. In Fluids 2000 Conference and Exhibit, Denver, CO, 2000.

[16] Lumley JL. The structure of inhomogeneous turbulence Atmospheric Turbulence and Wave Propagation, 1967; 166-178.

[17] Holmes P, Lumley JL, Berkooz G. Turbulence, Coherent Structures, Dynamical Systems and Symmetry. Cambridge University Press, 1996.

[18] Moin P, Moser RD. Characteristic-eddy decomposition of turbulence in a channel. Journal of Fluid Mechanics Digital Archive 2006; 200:471-509.

[19] Lieu T, Farhat C, Lesoinne M. Reduced-order fluid/structure modeling of a complete aircraft configuration. Computer methods in applied mechanics and engineering 2006; 195:5730-5742.

[20] Coelho RF, Breitkopf P, Knopf-Lenoir C. Mode reduction for multidisciplinary optimizationapplication to a 2D wing. Structural Multidisciplinary Optimization 2008; 37:29-48.

[21] Chatterjee A. An introduction to the proper orthogonal decomposition. Current Science 2000; 78(7):808-817.

[22] Sirovich L. Turbulence and the dynamics of coherent structures. I- Coherent structures. IISymmetries and transformations. III- Dynamics and scaling. Quarterly of Applied mathematics 1987; 45:561-571.

[23] Stein M. Large sample properties of simulations using Latin hypercube sampling. Technometrics 1987; pages 143-151.

[24] McKay MD. A comparison of three methods for selecting values of input variables in the analysis of output from a computer code. Technometrics 2000; pages 55-61.

[25] Levin D. The approximation power of moving least squares. Mathematics of Computation 1998; 67(224):1517-1531.

[26] Nealen A. An as-short-as-possible introduction to the least squares, weighted least squares and moving least squares methods for scattered data approximation and interpolation. URL: http://www.nealen.com/projects, 2004. 
[27] Wall WA, Ramm E. Fluid-structure interaction based upon a stabilized (ALE) finite element method. 1998.

[28] Hübner B, Walhorn E, Dinkler D. A monolithic approach to fluid-structure interaction using space-time finite elements. Computer Methods in Applied Mechanics and Engineering 2004; 193:2087-2104. 